\title{
SPATIAL GRAPH AS CONNECTED SUM OF A PLANAR GRAPH AND A BRAID
}

\author{
VALERIY G. BARDAKOV AND AKIO KAWAUCHI
}

\begin{abstract}
In this paper we show that every finite spatial graph is a connected sum of a planar graph, which is a forest, i.e. disjoint union of finite number of trees and a tangle. As a consequence we get that any finite spatial graph is a connected sum of a planar graph and a braid. Using these decompositions it is not difficult to find a set of generators and defining relations for the fundamental group of compliment of a spatial graph in 3 -space $\mathbb{R}^{3}$.
\end{abstract}

\section{INTRODUCTION}

For studying classical links in three dimensional space $\mathbb{R}^{3}$ people use some presentations of links. For example, link diagrams on a plane, rectangular diagram of a link [3]-4], link as the closure of a braid, link as a plat [1, and so on.

Theory of spatial graphs is a generalization of link theory in three dimensional space. L. Kauffman [7] defined two types of equivalence relations on the set of spatial graphs and prove some analogs of Reidemeister theorem. Also, he associated a collection of knots and links to a spatial graph that gives computable invariants for spatial graphs. Other invariants were constructed in [9] (see also [8]).

K. Kanno, K. Taniyama [6] for every oriented spatial graph found a braid presentation. This result is a generalization of Alexander's theorem.

In the present paper we suggest some other presentations of a spatial graph. At first we prove that every finite spatial graph is a connected sum of a planar graph, which is a forest, i.e. disjoint union of finite number of trees and a tangle. Using the Alexander theorem, we prove that this graph is a connected some of a forest and a braid (braid decomposition of the spatial graph). Also, we construct another type decomposition without using the Alexander theorem. We prove that any spatial graph is a connected sum of a forest and a plat-braid that is some symbiosis of a plat and a braid (plat decomposition of the spatial graph).

Date: November 10, 2021.

2000 Mathematics Subject Classification. Primary 57M25; Secondary 57M15.

Key words and phrases. Spatial graph, planar graph, tangle, braid, fundamental group of spatial graph.

The authors gratefully acknowledge the support of the grant RNF-16-11-10073. Also they thank Vera Gorbunova, who drew pictures for the article. 
Using these decompositions it is not difficult to find a set of generators and defining relations for the fundamental group of compliment of a spatial graph in 3-space $\mathbb{R}^{3}$ (see Section 4).

\section{Combinatorial GRAPHS AND SPATiAL GRAPHS}

A combinatorial graph $\mathcal{G}$ consists of a four $(V, E, i, t)$, where $V$ is the set of vertices, $E$ is the set of edges, $i, t: E \rightarrow V$ are two functions, $i(e)$ is called the beginning of $e$ and $t(e)$ is called the end of e. We write $V(\mathcal{G})$ for the vertices of $\mathcal{G}$ and $E(\mathcal{G})$ for the edges of $\mathcal{G}$ when necessary. If $i(e)=t(e)$, then $e$ is called a loop. A graph $\mathcal{G}$ is called finite if $V$ and $E$ are finite. A combinatorial graph can contains multiple edges, but we will assume that $\mathcal{G}$ has no vertices of degrees 0 and 1 .

A spatial graph $\Gamma$ is a geometric realization of a combinatorial graph $\mathcal{G}$ in $\mathbb{R}^{3}$, that is an injective map $\Gamma \rightarrow \mathbb{R}^{3}$ under which $V$ goes to a set of distinct points and any $e$ in $E$ goes to a topological interval $L_{e}$ that is a topological space homeomorphic to the close interval $[0,1]$ in the set of real numbers $\mathbb{R}$, which is beginning in the image of the vertex $i(e)$ and ending in the image of the vertex $t(e)$ if $i(e) \neq t(e)$; if $i(e)=t(e)$, then $L_{e}$ homeomorphic to the circle. The topological interval $L_{e}$ can meet with the images of $V$ only in the beginning or ending points and two different topological intervals do not intersect at internal points. For examples, if $\mathcal{G}$ contains one vertex $v$ and one edge $e$, where $i(e)=t(e)$, then the set of spatial graphs is the set of knots in $\mathbb{R}^{3}$. Two spatial graphs $\Gamma$ and $\Gamma^{\prime}$ are equivalent if there is an orientation-preserving homeomorphism $h: \mathbb{R}^{3} \longrightarrow \mathbb{R}^{3}$ sending $\Gamma$ onto $\Gamma^{\prime}$. A fundamental topological problem (equivalence decision problem) on spatial graphs is:

Problem 1. By an effective method, decide whether or not two given spatial graphs of a combinatorial graph are equivalent.

A diagram $D_{\Gamma}$ of a spatial graph $\Gamma$ is a regular projection of $\Gamma$ to a plane in $\mathbb{R}^{3}$. Equivalence relation for spatial graphs is generated by a set of local moves that generalize the Reidemeister moves for diagrams of knots. L. Kauffman [7] added to the usual list of Reidemeister moves two moves involving a vertex (moves IV and V in Figure 1). 
I

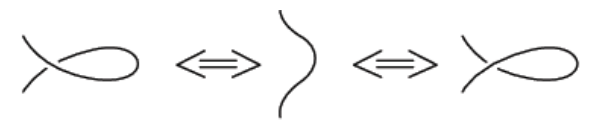

II

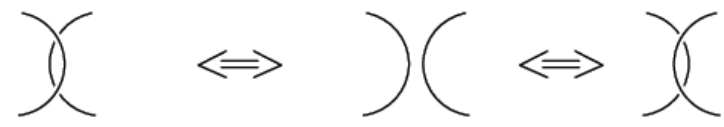

III

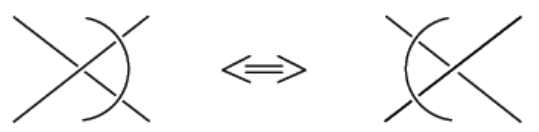

IV
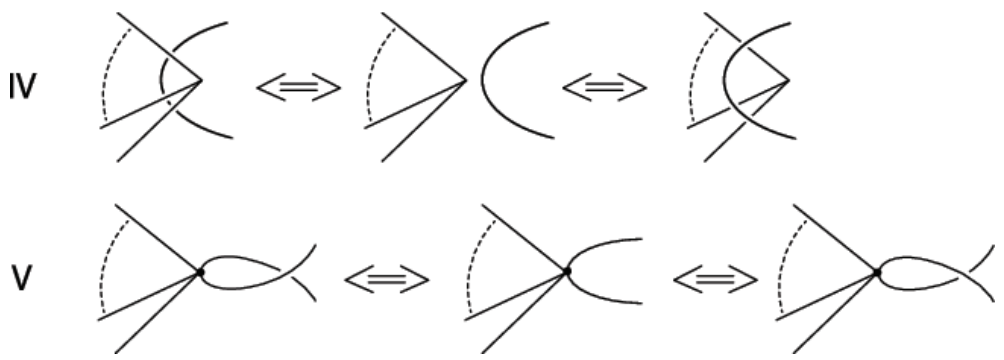

Fig. 1. Reidemeister moves

Moves IV allows an edge to slide under or over a bundle of strands at a vertex. Move $\mathrm{V}$ allows any two adjacent (in the planar diagram) strands at a vertex to twist around one another. This is the basic topological vertex move: two strands at the vertex can twist without affecting the other strands. L. Kauffman proved that two spatial graphs $\Gamma$ and $\Gamma^{\prime}$ are equivalent if and only if any diagram $D$ of $\Gamma$ is deformed into any diagram $D^{\prime}$ of $\Gamma^{\prime}$ by a finite sequence of the generalized Reidemeister moves $\mathrm{I}-\mathrm{V}$.

Recall that a connected non-empty graph is called a tree if it does not have cycles and multiples edges, a connected non-empty graph is called a forest if it is a disjoint union of trees. It is evident, that every forest is a planar graph.

We say that a spatial graph $\Gamma$ is a connected sum of two spatial subgraphs $\Gamma_{1}$ and $\Gamma_{2}$ and write $\Gamma=\Gamma_{1} \sharp \Gamma_{2}$, if there is a 3 -ball $B \subset \mathbb{R}^{3}$ such that its boundary $S^{2}=\partial B$ does not contain vertices of $\Gamma$, the intersection $S^{2} \cap \Gamma$ is a finite number of points, the closure of $\left(\mathbb{R}^{3}-B\right) \cap \Gamma$ is equal to $\Gamma_{1}$ and the closure of $B \cap \Gamma$ is equal to $\Gamma_{2}$.

\section{BRAID DECOMPOSITION}

The main result of the present section is

Theorem 3.1. Let $\Gamma$ be a finite spatial graph in $\mathbb{R}^{3}$. Then there are a forest $T_{0}$ and a braid $\beta$ such that $\Gamma=T_{0} \sharp \beta$.

For illustration of this theorem see Fig. 2, where a forest is in the left box and a braid in the right box. 


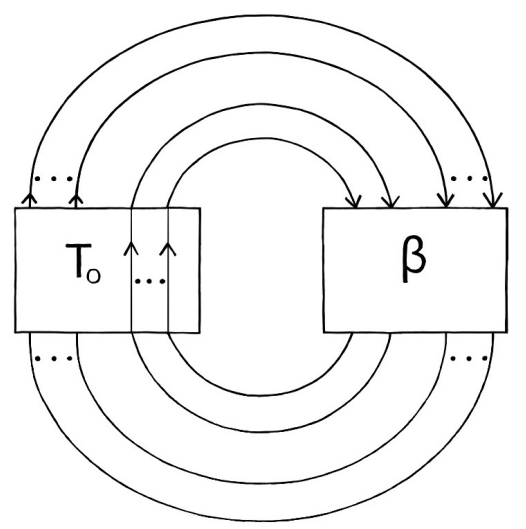

Fig. 2. Spatial graph as a connected sum of forest and braid

We prove the theorem for connected graphs. The general case is similar. Let $\Gamma$ be a finite connected spatial graph in $\mathbb{R}^{3}$. Denote by $V(\Gamma)$ the set of all vertices of $\Gamma$ and by $E(\Gamma)$ the set of all edges of $\Gamma$. Take some maximal tree $T$ of $\Gamma$. By the definition of maximal tree, the set of $V(T)$ is equal to the set $V(\Gamma)$ and the set of edges $E(T)$ is a subset of $E(\Gamma)$. If $E(T)=E(\Gamma)$, then $\Gamma=T$ and the theorem is true. Hence, we shall assume that $E(T) \neq E(\Gamma)$. We call the edges in $E(T)$ by tree edges and the edges in $E(\Gamma) \backslash E(T)$ by spatial edges. The set $V(T)$ is the disjoint union of two subsets: $V(T)=I(T) \sqcup S(T)$, where $I(T)$ is the set of inner vertices, i.e. vertices which are incident only tree edges; $S(T)$ is the set of spatial vertices, i.e. vertices which are incident some spatial edges. Every spatial edge $e$ has the initial vertex $i(e)$ and the terminal vertex $t(e)$, which lie in $S(T)$.

Transform the graph $\Gamma$ in $\mathbb{R}^{3}$ by the such manner that the tree $T$ lies on the plane $x O y$ and all spatial edges lie in the subspace $z \geq 0$ (see Fig. 3). To do it we can consider a diagram of $\Gamma$ and by using the generalized Reidemeister moves transform it to the needed diagram.

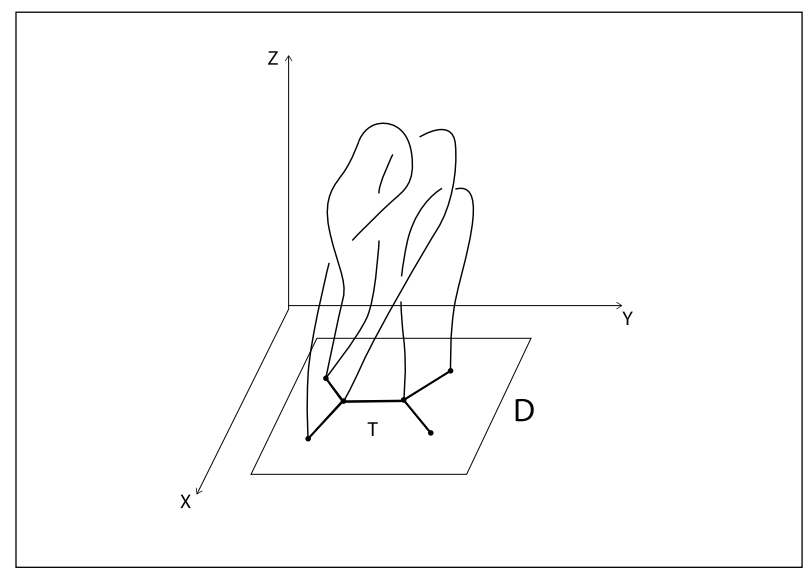

Fig. 3. A maximal tree $T$ on the plane

We assume that the tree $T$ lies inside of some rectangle $D$ those sides are parallel to the axes $O x$ and $O y$. Take a some vertex in $S(T)$ and call this vertex by the initial 
vertex and shift $T$ to a position where this vertex lies in the upper part of $D$ with respect to the $y$-coordinate; the vertices which are connected to the initial vertex by some edge in $E(T)$ lie in a lower level with respect to $y$-coordinate; the vertices which are connected to the vertices of the second level by some edge in $E(T)$ lie in the third level and so on. See the upper picture of Fig. 3, where vertex $a$ is the initial vertex and lies in the first level, vertices $b, c$ and $d$ lie in the second level and so on. Using induction by the number of levels transform the graph $\Gamma$ to a graph in which all the vertices in $V(T)$ lie in the middle line of $D$. See the lower picture in Fig. 4, where we transformed the vertex $a$ from the first level to the second level.

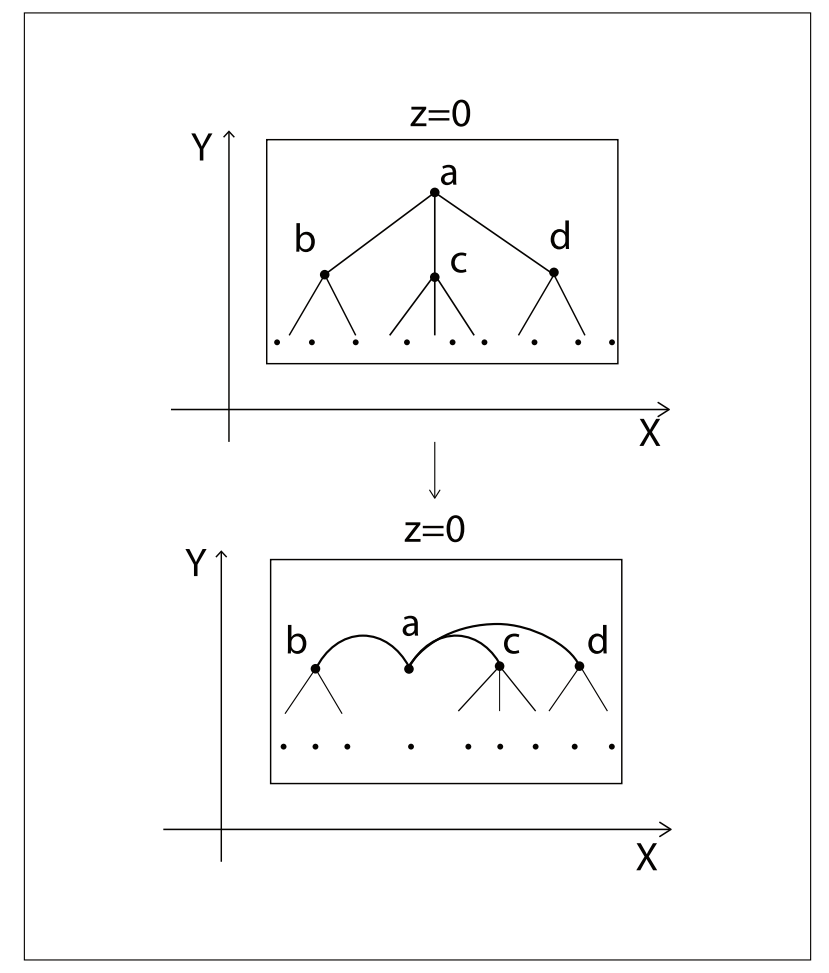

Fig. 4. Transformation of the tree

For every vertex $v \in S(T)$ construct a square $V_{v}=V_{v}^{0}$ on the plane $x O y$, such that $v$ is the center of this square and the sides of this square are parallel to the axes $O x$ and $O y ; V_{v}$ does not contain the other vertices of $T$. Let $V_{v}^{1}$ be the orthogonal projection of $V_{v}$ onto the plane $z=1$. Move the spatial edges for which $v$ is the initial vertex to the position in which these edges intersect the upper side (side with larger $y$-coordinate) of $V_{v}^{1}$ and the spatial edges for which $v$ is the terminal vertex to the position in which these edges intersect the bottom side (side with smaller $y$-coordinate) of $V_{v}^{1}$ (see Fig. 5). 


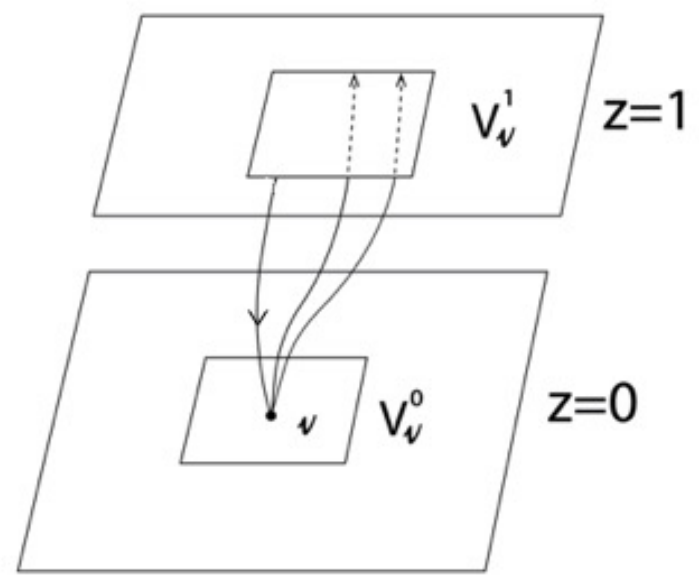

Fig. 5. Spatial edges in the neighborhood of a vertex

Do this for all spatial vertices to get in the plane $z=1$ a picture as in Fig. 6 . Denote by $\bar{T}$ the closure of the intersection of $\Gamma$ with the subspace $z \leq 1$. We see that $\bar{T}$ is a tree. Consider the subspace $z \geq 1$. The intersection of this subspace with $\Gamma$ is the union of strings.

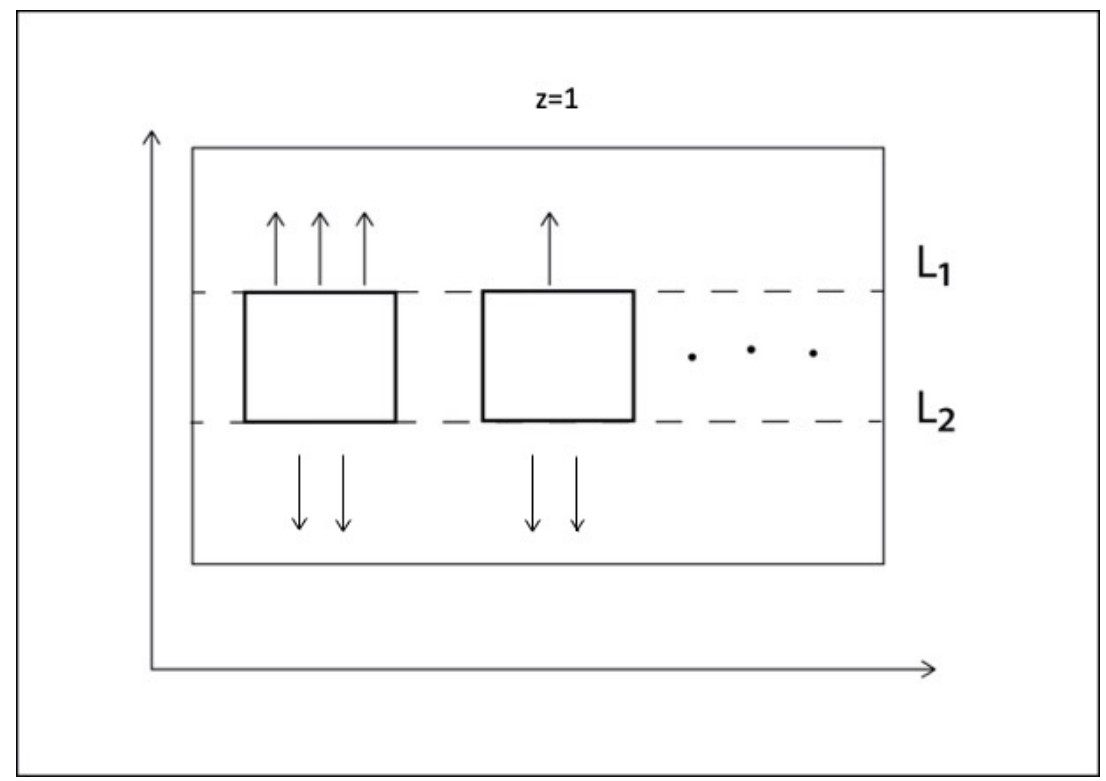

Fig. 6. Rectangles for the vertices 
We can put these strings in the box $D_{1} \times[0,1]$, where, $D_{1}$ be a rectangle in the plane $z=1$ with sides that are parallel to the coordinate lines $O x$ and $O y$. We get some $(m, m)$-tangle $\bar{\beta}$ (see Fig. 7 ), where $m$ is the set of spatial edges in $\Gamma$. Hence, $\Gamma$ is the connected sum of $\bar{T}$ and $\bar{\beta}$. Tangle $\bar{\beta}$ does not contain free components and each his edge is stating at the upper side and ending at the bottom side of the parallelepiped $D_{1} \times[0,1]$.

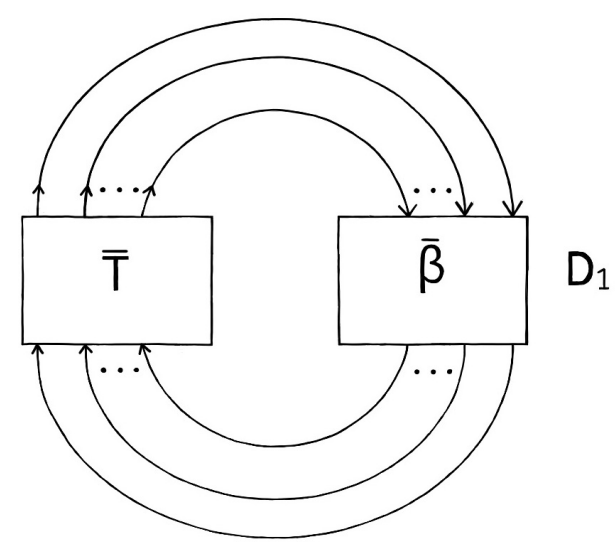

Fig. 7. Tree and tangle

Hence, we have proven

Proposition 3.2. Any finite spatial graph $\Gamma$ is a connected sum of a forest $\bar{T}$ and a tangle $\bar{\beta}$.

In this decomposition the forest $\bar{T}$ contains $2 m$ edges, where $m=|E(\Gamma)|-|E(T)|$, which do not lie in the maximal forest $T$ of $\Gamma$. From these edges $m$ have beginning vertex of valence 1 , denote them $a_{1}, a_{2}, \ldots, a_{m}$ and will call incoming edges. Other $m$ edges have terminating vertex of valence 1 , denote them $b_{1}, b_{2}, \ldots, b_{m}$ and will call outgoing edges. The tangle $\bar{\beta}$ is $(m, m)$-tangle that is a tangle with $m$ incoming strings and $m$ outgoing strings. To get the spatial graph $\Gamma$ we must gluing the outgoing edges of the forest with the incoming strings of the tangle and the outgoing strings of the tangle with the incoming edges of $\bar{T}$.

Using Proposition 3.2 we can prove Theorem 3.1.

Proof. We assume that the spatial graph $\Gamma$ is connected and by Proposition 3.2 it is the connected sum of the tree $\bar{T}$ and the tangle $\bar{\beta}$. Using the same idea as in the proof of the Alexander theorem [1, Chapter 2.1], we can present $\bar{\beta}$ as a connected sum of a braid $\beta$ and some number of unknotted and unlinked arcs. Adding these arcs to the tree $\bar{T}$ we get the forest $T_{0}$ and the decomposition of $\Gamma$ as the connected sum of $T_{0}$ and the braid $\beta$ (see Fig. 2).

\section{Plat DeComposition}

Any link can be present as a closure of a braid or as a plat (see [1]). We introduce some object that is a symbiosis of a plat and a braid. Let $k, m$ be non-negative 
integer numbers and

$$
I^{3}=\left\{(x, y, z) \in \mathbb{R}^{3} \mid 0 \leq x, y, z \leq 1\right\}
$$

be the cub with the side 1 . On the upper side take $2 k+m$ points $P_{1}, P_{2}, \ldots$, $P_{2 k+m}$, which lie in the plane $y=1 / 2$ and the point with bigger index has bigger $x$-coordinate. On the bottom side also take $2 k+m$ points $Q_{1}, Q_{2}, \ldots, Q_{2 k+m}$, which also lie in the plane $y=1 / 2$ and the point with bigger index has bigger $x$-coordinate.

The $m$-component $k$-plat-braid or simply $(k, m)$-plat-braid $L_{k, m}=L_{1} \sqcup L_{2} \sqcup \ldots \sqcup L_{m}$ is the disjoint union of $m$ topological intervals, which are images of $m$ segments $[0,1]$ into the 3 -space $\mathbb{R}^{3}$ and the following conditions hold:

1) all points $P_{i}$ and $Q_{i}$ lie on $L_{k, m}$ and

$$
L_{k, m} \cap \partial I^{3}=\left\{P_{1}, P_{2}, \ldots, P_{2 k+m}, Q_{1}, Q_{2}, \ldots, Q_{2 k+m}\right\}
$$

2) $\partial L_{k, m}$ lie in $\mathbb{R}^{3} \backslash I^{3}$, the first intersection point of $L_{i}$ and $I^{3}$ is $P_{i}$ and the last intersection point of $L_{i}$ and $I^{3}$ is $Q_{i}$ for all $i \in\{1,2, \ldots, m\}$.

3) Any pair of points $\left\{P_{j}, P_{j+1}\right\},\left\{Q_{j}, Q_{j+1}\right\}, j=1,2, \ldots, k-1$ is connected by some arc which is a part of $L_{k, m}$.

4) If we forget about orientation of $L_{k, m}$, then the intersection $L_{k, m} \cap I^{3}$ is an $2 k+m$-braid $\gamma$.

For example of $(2,2)$-plat-braid see Figure 8.

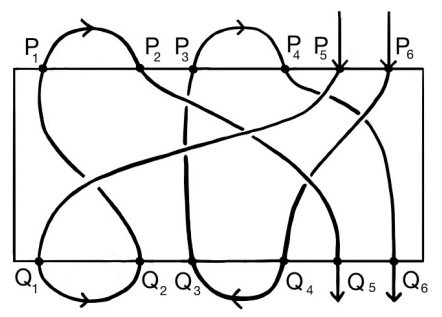

Fig. 8. Example of $(2,2)$-plat-braid

The main result of the present section is

Theorem 4.1. Let $\Gamma$ be a finite spatial graph with $n$ connected components in $\mathbb{R}^{3}$. Then there are a forest $\bar{T}$, which is a disjoint union of $n$ trees and a plat-braid $\gamma$ such that $\Gamma=\bar{T} \sharp \gamma$. If $\Gamma$ is a connected spatial graph, then the forest $\bar{T}$ is a tree.

For illustration of this theorem see Figure 9. We shall call the decomposition of $\Gamma$ from this theorem by plat decomposition 


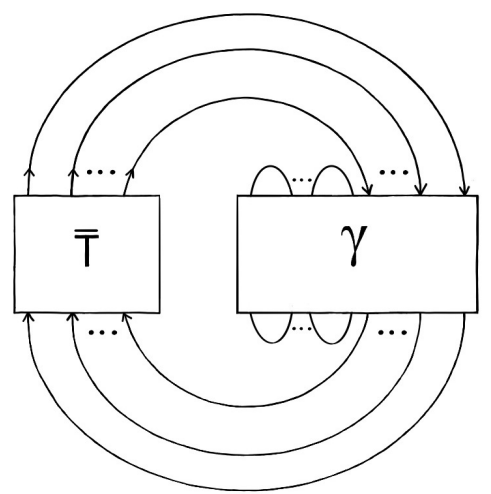

Fig. 9. Spatial graph as decomposition of a forest and a plat-braid

Proof. We assume that the spatial graph $\Gamma$ is connected and by Proposition 3.2 it is the connected sum of the tree $\bar{T}$ and the tangle $\bar{\beta}$.

We shall transform $\bar{\beta}$ to get some plate-braid. We can assume that under the projection of $\bar{\beta}$ onto the plane $z=1$ all crossings lie inside $D_{1}$ and that this projection is regular projection. This projection has finite number of local maximums and local minimums with respect to $y$-coordinate. We can check that the number of the local maximums is equal to the number of local minimums. Denote by $m_{1}, m_{2}, \ldots, m_{k}$ the points of local maximums and by $l_{1}, l_{2}, \ldots, l_{k}$ the points of local minimums (see Fig. 10). Using isotopy we will move the arcs with maximum to the upper side of $D_{1}$ and the arcs with minimum to the bottom side of $D_{1}$.

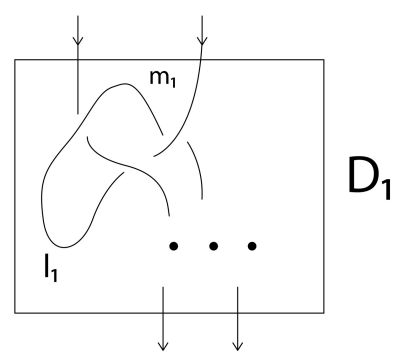

Fig. 10. Local maximum and local minimum

Moving this arcs until they will be outside of $D_{1}$. Using the transformations as in Figure 11 we can assume that outside of $D_{1}$ all arcs with maximum lie on the left side from the edges which go inside the rectangle and all arcs with minimum lie on the left side from the edges which go outside the rectangle. 

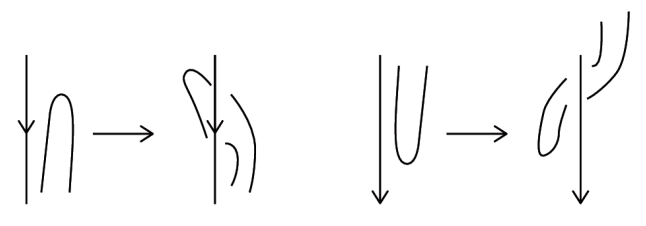

Fig. 11. Moving of arcs with local maximum and minimum

At the end we transform the $(m, m)$-tangle $\bar{\beta}$ to the $(k, m)$-plat-braid $\gamma$ in which the number of connected components is $m=|E(\Gamma)|-|E(T)|$ (see Fig. 12).

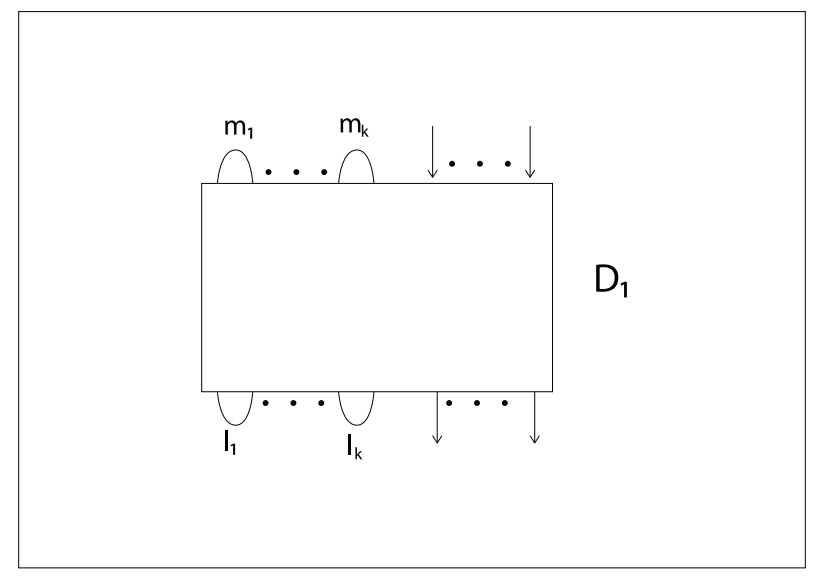

Fig. 12. The $(k, m)$-plat-braid $\gamma$

Take the decomposition $\Gamma=\bar{T} \sharp \bar{\beta}$, constructed in Proposition 3.2, cut the tangle $\bar{\beta}$ and paste the $(k, m)$-plat-braid $\gamma$, then we get the plat decomposition $\Gamma=\bar{T} \sharp \gamma$.

\section{Some APplications}

5.1. Braid index. Using the braid decomposition we can introduce some invariant of spatial graph. The braid index bi $\left(T_{0} \sharp \beta\right)$ of the braid decomposition $\Gamma=T_{0} \sharp \beta$ is the number of strings in the braid $\beta$. The braid index bi $(\Gamma)$ of the spatial graph $\Gamma$ is the minimum $b i\left(T_{0} \sharp \beta\right)$ by all possible braid decompositions $\Gamma=T_{0} \sharp \beta$.

It is not difficult to see that $b i(\Gamma)$ is an invariant of $\Gamma$.

Question 1. Are there some connections of bi $(\Gamma)$ with other invariants of $\Gamma$ ? 
5.2. Groups of spatial graphs. Let $\Gamma$ be a spatial graph, then its group $G_{\Gamma}$ is the fundamental group of the complement $\Gamma$ in 3-space: $G_{\Gamma}=\pi_{1}\left(\mathbb{R}^{3}-\Gamma\right)$. We can find the set of generators and defining relations for this group. To do it, consider the regular projection $D_{\Gamma}$ of $\Gamma$ into the plane and remove all vertices of valence $\geq 3$. Denote the connected components of this diagram by $x_{1}, x_{2}, \ldots, x_{s}$ this is the generating set of $G_{\Gamma}$. Defining relations can be two types. Any crossing of $D_{\Gamma}$ corresponds defining relation as in Fig. 13.
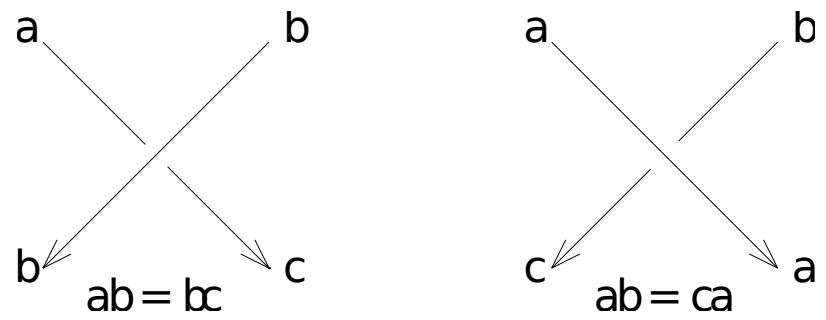

Fig. 13. Relations in the crossings

Any vertex of $D_{\Gamma}$ corresponds defining relation as in Figure 14.

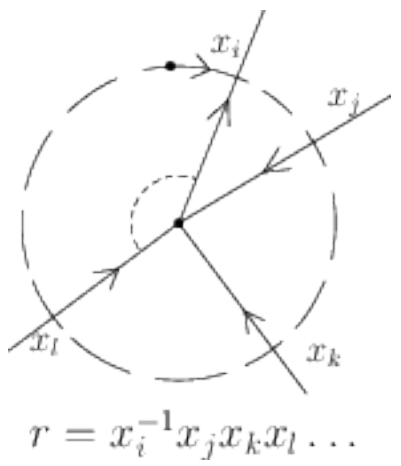

Fig. 14. Relation in the vertex

For simplicity we will consider the case when $\Gamma$ is a connected graph.

Suppose we have decomposition $\Gamma=\bar{T} \sharp \bar{\beta}$, constructed in Proposition 3.2. To find $G_{\Gamma}$, define a group $G_{\bar{T}}$ and a group $G_{\bar{\beta}}$. Suppose that a 3 -ball $B$ contains the forest $\bar{T}$ and $\bar{\beta}=\Gamma \backslash(B \cap \bar{T})$. Then

$$
G_{\bar{T}}=\pi_{1}(B \backslash \bar{T}), G_{\bar{\beta}}=\pi_{1}\left(\left(\mathbb{R}^{3} \backslash B\right) \backslash \bar{\beta}\right) .
$$

To find $G_{\Gamma}$ note that $G_{\bar{T}}=G_{\overline{T_{1}}}$, where $\overline{T_{1}}$ is a tree that is gotten from $\bar{T}$ by contracting of the maximal tree $T_{0}$ into a vertex. Hence, $\overline{T_{1}}$ contains one vertex and $m$ incoming edges $a_{1}, a_{2}, \ldots, a_{m}$ and $m$ outgoing edges $b_{1}, b_{2}, \ldots, b_{m}$ and

$$
G_{\overline{T_{1}}}=\left\langle a_{1}, a_{2}, \ldots, a_{m}, b_{1}, b_{2}, \ldots, b_{m} \| a_{1} a_{2} \ldots a_{m} b_{m}^{-1} b_{m-2}^{-1} \ldots b_{1}^{-1}=1\right\rangle .
$$

Let $b_{1}^{\prime}, b_{2}^{\prime}, \ldots, b_{m}^{\prime}$ be the set of incoming strings in $\bar{\beta}$ and $a_{1}^{\prime}, a_{2}^{\prime}, \ldots, a_{m}^{\prime}$ be the set of outcoming strings in $\bar{\beta}$. Then the group $G_{\bar{\beta}}$ contains elements

$$
a_{1}^{\prime}, a_{2}^{\prime}, \ldots, a_{m}^{\prime}, b_{1}^{\prime}, b_{2}^{\prime}, \ldots, b_{m}^{\prime}
$$


Suppose that $G_{\bar{\beta}}$ is defined by a set of generators $\mathcal{X}$ and the set of relations $\mathcal{R}$, i.e.

$$
G_{\bar{\beta}}=\langle\mathcal{X} \| \mathcal{R}\rangle \text {. }
$$

Then from Van Kampen theorem follows

Theorem 5.1. The group $G_{\Gamma}$ is generated by elements

$$
\mathcal{X}, a_{1}, a_{2}, \ldots, a_{m}, b_{1}, b_{2}, \ldots, b_{m}
$$

and is defined by the relations

$$
\begin{gathered}
\mathcal{R}, a_{1} a_{2} \ldots a_{m} b_{m}^{-1} b_{m-2}^{-1} \ldots b_{1}^{-1}=1, b_{1}=b_{m}^{\prime}, b_{2}=b_{m-1}^{\prime}, \ldots, b_{m}=b_{1}^{\prime}, \\
a_{1}=a_{m}^{\prime}, a_{2}=a_{m-1}^{\prime}, \ldots, a_{m}=a_{1}^{\prime} .
\end{gathered}
$$

In particular, if $\Gamma$ is a link then we can decompose it in the form $\Gamma=\bar{T} \sharp \bar{\beta}$, where $\bar{T}$ is the disjoint union of $m$ edges $b_{1}=a_{1}, b_{2}=a_{2}, \ldots, b_{b}=a_{m}$ and we have

Corollary 5.2. The group $G_{\Gamma}$ is generated by elements $\mathcal{X}$ and is defined by the relations

$$
\mathcal{R}, a_{1}^{\prime}=b_{1}^{\prime}, a_{2}^{\prime}=b_{2}^{\prime}, \ldots, a_{m}^{\prime}=b_{m}^{\prime}
$$

In [9] was considered conception of unknotted spatial graph. In our terms we can reformulate it by the following manner. A spatial graph $\Gamma$ is called unknotted if there is a decomposition $\Gamma=\bar{T} \sharp \bar{\beta}$ into a connection sum of a forest and a tangle, where the tangle $\bar{\beta}$ is monotone. A tangle is called monotone if it has a monotone diagram. A strand in a diagram of a tangle is called monotone if a point going along the oriented strands meets first the upper crossing point at every crossing point. A diagram of a tangle is called monotone if every its strand is monotone and there is some ordering of strands such that the strand with number $i$ is upper than the strands with number $j$ for $i<j$.

As corollary of Theorem 5.1 we get

Corollary 5.3. Suppose that a finite connected spatial graph $\Gamma$ has a decomposition $\Gamma=\bar{T} \sharp \bar{\beta}$. Then

1) if $\bar{\beta}$ is a $(1,1)$-tangle, then $G_{\Gamma}$ is isomorphic to the group $G_{K}$ of the knot $K$, which is the closure of the tangle $\bar{\beta}$;

2) if $\bar{\beta}$ is a monotone tangle with $k$ strands. Then $G_{\Gamma}$ is the free group of rank $k$.

Proof. 1) In this case $\bar{T}$ is a line segment with a finite set of vertexes. If we compress this segment into a vertex, then our spatial graph becomes a knot $K$ that is the closure of $\bar{\beta}$.

2) Follows from [9], where was proved the following assertion: An unknotted connected spatial graph is equivalent to a trivial bouquet of circles after the edge contraction of a maximal tree. In particular, the group of unknotted connected spatial graph is free. 


\section{Conclusion Remarks}

Of cause, the decomposition of the spatial graph $\Gamma$ in Proposition 3.2 is not unique, because there are different possibilities in the choice of the maximal forest. We can formulate

Conjecture 1. Let $\Gamma$ and $\Gamma^{\prime}$ are two finite spatial graphs, which correspond to some combinatorial graph $\mathcal{G}$. They are equivalent if and only if there are decompositions $\Gamma=\bar{T} \sharp \bar{\beta}$ and $\Gamma^{\prime}=\overline{T^{\prime}} \sharp \overline{\beta^{\prime}}$ into the connected sum of a forest and a tangle such that the forest $\bar{T}$ is equivalent to the forest $\overline{T^{\prime}}$ (as spatial graphs) and the tangle $\bar{\beta}$ is equivalent to the tangle $\overline{\beta^{\prime}}$.

When we have decomposition of two spatial graphs in the connected sum of a forest and a braid, then the equivalence decision problem is more complicated and we formulate

Question 2. Let $\Gamma=T_{0} \sharp \beta$ and $\Gamma=T_{0}^{\prime} \sharp \beta^{\prime}$ be two decompositions of some finite spatial graph $\Gamma$. How are they related? Is it possible to prove some analog of Markov theorem for spatial graphs?

We know the following problem: for the spatial graph $\Gamma$ find all links, which can be embedding in $\Gamma$. A constituent link of a spatial graph $\Gamma$ is a link contained in $\Gamma$. Conway and Gordon 2 proved that every spatial 6-complete graph $K_{6}$ contains a non trivial constituent link and every spatial 7-complete graph $K_{7}$ contains a non trivial constituent knot. Is it possible to prove these results, using a decomposition of a spatial graph, constructed in the present paper?

We know the construction of a knot quandle.

Question 3. Is it possible to define for any spatial graph $\Gamma$ a quandle $Q_{\Gamma}$ such that, if $\Gamma^{\prime}$ is equivalent to $\Gamma$, the $Q_{\Gamma}$ is isomorphic to $Q_{\Gamma^{\prime}}$ ?

\section{REFERENCES}

[1] J. S. Birman, Braids, links and mapping class group, Princeton-Tokyo: Univ. press, 1974.

[2] J. H. Conway, C. McA. Gordon, Knots and links in spatial graphs, J. Graph Theory, 7, no. 4 (1983), 445-453.

[3] P. R. Cromwell, Embedding knots and links in an open book I: Basic properties, Topology and its Applications 64 (1995), 37-58.

[4] P. R. Cromwell and I. J. Nutt, Embedding knots and links in an open book II: bounds on arc index, Math. Proc. Cambridge Philos. Soc. 119:2 (1996), 309- 319.

[5] R. H. Crowell, R. H. Fox, Introduction to knot theory. Based upon lectures given at Haverford College under the Philips Lecture Program Ginn and Co., Boston, Mass. 1963 x+182 pp.

[6] K. Kanno, K. Taniyama, Braid presentation of spatial graphs, Tokyo J. Math., 33, no. 2 (2010), 509-522.

[7] L. H. Kauffman, Invariants of graphs in three space, Trans. Amer. Math. Soc., 1989, 311, 697-710. 
[8] A. Kawauchi, A survey of knot theory. Translated and revised from the 1990 Japanese original by the author. Birkhauser Verlag, Basel, 1996. xxii+420 pp.

[9] A. Kawauchi, On transforming a spatial graph into a plane graph, in: Statistical Physics and Topology of Polymers with Ramifications to Structure and Function of DNA and Proteins, Progress of Theoretical Physics Supplement, no. 191 (2011), 225-234.

Sobolev Institute of Mathematics, Novosibirsk State University, Novosibirsk 630090, Russia

Novosibirsk State Agrarian University, Dobrolyubova street, 160, Novosibirsk, 630039, RUSSIA

Tomsk State University, PR. Lenina, 36, Tomsk, 634050, Russia.

E-mail address: bardakov@math.nsc.ru

Department of Mathematics, Osaka City University Sugimoto, Sumiyoshi-ku, OsAKA 558-8585, JAPAN

E-mail address: kawauchi@sci.osaka-cu.ac.jp 